\title{
Jesus Handbuch
}

\author{
Hrsg. v. Jens Schröter u. Christine Jacobi, unter Mitarbeit v. Lena Nogossek
}

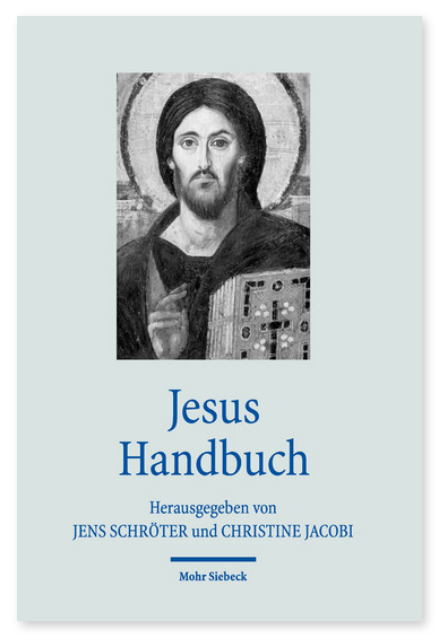

2017. XIII, 685 Seiten. HdTh

ISBN 978-3-16-155409-4 DOI 10.1628/978-3-16-155409-4 eBook PDF $49,00 €$

ISBN 978-3-16-153853-7 fadengeheftete Broschur 49,00€
Das Jesus Handbuch präsentiert die Forschung zum historischen Jesus und Rezeptionen seiner Person auf dem aktuellen Stand der internationalen Diskussion. Es gibt einen Überblick über Deutungen Jesu in der Christentumsgeschichte von den Anfängen bis zum Beginn des 21. Jahrhunderts. Behandelt werden des Weiteren Wirken, Lehre und Geschick Jesu in ihrem religiösen, sozialen und politischen Kontext. Dabei werden aktuelle geschichtswissenschaftliche Diskurse, archäologische Forschungen sowie Deutungen Jesu in neutestamentlichen und weiteren frühchristlichen Texten berücksichtigt. Schließlich richtet sich der Blick auf wichtige Wirkungen Jesu im frühen Christentum. Geboten wird somit ein Überblick über Person und Wirken Jesu sowie Deutungen seiner Person aus unterschiedlichen Perspektiven. An dem Handbuch mitgewirkt haben renommierte Forscherinnen und Forscher aus verschiedenen Kontexten. Das Handbuch bietet deshalb zugleich einen repräsentativen Überblick über die gegenwärtige Jesusforschung.

Inhaltsübersicht

A.Jens Schröter/Christine Jacobi: Einleitung

B. Geschichte der historisch-kritischen Jesusforschung

Jens Schröter/Christine Jacobi: Einführung - Martin Ohst: Der irdische Jesus in der antiken, mittelalterlichen und reformatorischen Frömmigkeit und Theologie - Albrecht Beutel: Das 18. Jahrhundert als Entstehungskontext der kritischen Theologie - Eckart David Schmidt: Die kritische Geschichtswissenschaft des späten 18. und 19. Jahrhunderts und ihre Auswirkung auf die Jesusforschung - John S. Kloppenborg: Die Einführung des Mythosbegriffs in die Jesusforschung und die Entstehung der Zweiquellentheorie - James Carleton Paget: Das »Gottesreich« als eschatologisches Konzept: Johannes Weiß und Albert Schweitzer - Reinhard von Bendemann: Historischer Jesus und kerygmatischer Christus - Cilliers Breytenbach: Die literarischen Entwürfe der Evangelien und ihr Verhältnis zum historischen Jesus - Chris Keith: Die Evangelien als »kerygmatische Erzählungen« über Jesus und die »Kriterien« in der Jesusforschung - David du Toit: Die »Third Quest for the Historical Jesus« - Jens Schröter: Der »erinnerte Jesus«: Erinnerung als geschichtshermeneutisches Paradigma der Jesusforschung

\section{Das historische Material}

I. Jens Schröter/Christine Jacobi: Einführung

II. Literarische Zeugnisse

1. Christliche Texte

John S. Kloppenborg: Die Synoptischen Evangelien, die Logienquelle (Q) und der historische Jesus - Jörg Frey:

Johannesevangelium - Christine Jacobi: Sonstige Schriften des Neuen Testaments - Simon Gathercole: Außerkanonische Schriften als Quellen für den historischen Jesus?

2. Nichtchristliche Texte

Steve Mason: Griechische, römische und syrische Quellen über Jesus - Steve Mason: Jüdische Texte: Flavius Josephus III. Nichtliterarische Zeugnisse

Jürgen K. Zangenberg: Archäologische Zeugnisse - Jürgen K. Zangenberg: Inschriften und Münzen

\section{Leben und Wirken Jesu}

I. Jens Schröter/Christine Jacobi: Einführung

II. Politische Verhältnisse und religiöser Kontext

Danie/ R. Schwartz: Politische Verhältnisse: Römische Herrschaft, Herodes der Große, Antipas - Lutz Doering: Religiöser Kontext

III. Biographische Aspekte

Stephen Hultgren: Jesus: Herkunft, Familie, Geburt, Kindheit - Stephen Hultgren: Die Bildung und Sprache Jesu - Lutz Doering: Jesus im Judentum seiner Zeit (Die jüdische Prägung Jesu) - Jürgen K. Zangenberg: Galiläa und Umgebung als Wirkungsraum - Jürgen K. Zangenberg: Jerusalem und Judäa als Wirkungsraum IV. Öffentliches Wirken

1. Der soziale Kontext Jesu

Knut Backhaus: Jesus und Johannes der Täufer - James G. Crossley: Jesus im politischen und sozialen Umfeld seiner Zeit 2. Das Handeln Jesu

Joseph Verheyden: Jesus als Wanderprediger - Joseph Verheyden: Gründung einer Gemeinschaft: Ruf in die Nachfolge und die Bildung des Zwölferkreises - Hermut Löhr: Mahlgemeinschaften Jesu - Annette Weissenrieder: Heilungen Jesu - Bernd Kollmann: Exorzismen - Bernd Kollmann: Totenerweckungen und Naturwunder - Christiane Zimmermann: Frauen im Umfeld Jesu - Christiane Zimmermann: Jesus und das Volk - Darrel/ L. Bock/Jens Schröter: Jesu Perspektive auf Israel - Yair Furstenberg: Zöllner und Sünder als Adressaten des Wirkens Jesu - Martina Böhm: Jesu Verhältnis zu den Samaritanern 3. Die Reden Jesu / Das Lehren Jesu

Christine Gerber: Das Gottesbild Jesu und die Bedeutung der Vatermetaphorik - Craig Evans/Jeremiah J. Johnston: Gottesherrschaft - Ruben Zimmermann: Gleichnisse und Parabeln - Michael Wolter: Gerichtsvorstellungen Jesu - KarlHeinrich Ostmeyer: Das Beten Jesu, Vaterunser - Thomas Kazen: Jesu Interpretation der Tora - Martin Ebner: Jesus als Weisheitslehrer - Michael Wolter: Jesu Selbstverständnis

4. Das Ethos Jesu

Friedrich Wilhelm Horn: Nächstenliebe und Feindesliebe - Friedrich Wilhelm Horn: Besitz und Reichtum - Michael Labahn: Nachfolge, radikaler Verzicht, »a-familiäres« Ethos - Michael Labahn: Jesus als »Fresser und Weinsäufer «

5. Die Passionsereignisse 
Markus Tiwald: Einzug in Jerusalem, Tempelreinigung (Jesu Stellung zum Tempel) - Hermut Löhr: Das letzte Mahl Jesu - SvenOlav Back: Die Prozesse gegen Jesus - Sven-Olav Back: Kreuzigung und Grablegung Jesu

\section{E. Frühe Spuren von Wirkungen und Rezeptionen Jesu}

Jens Schröter/Christine Jacobi: Einführung - Christine Jacobi: Auferstehung, Erscheinungen, Weisungen des Auferstandenen Samuel Vollenweider: Frühe Glaubensbekenntnisse - David du Toit: Christologische Hoheitstitel - Markus Öhler: Ausbildung von Strukturen: Die Zwölf, Wandercharismatiker, Jerusalemer Urgemeinde und Apostel - Tobias Nicklas: Jesus in außerkanonischen Texten des 2. und 3. Jahrhunderts - Katharina Heyden/Rahel Schär: Bildliche Darstellungen Jesu bis ca. 500 n.Chr. - Ulrich Volp: Ethik (Bergpredigt)

Jens Schröter Geboren 1961; 1992 Promotion; 1996 Habilitation; Professur für Exegese und Theologie des Neuen Testaments sowie die antiken christlichen Apokryphen an der Theologischen Fakultät der Humboldt-Universität zu Berlin. https://orcid.org/0000-0001-7878-2709

Christine Jacobi Geboren 1979; Studium der Kunstgeschichte und der Ev. Theologie; 2014 Promotion; 2020 Habilitation; seit 2021 Vikarin in der Evangelischen Landeskirche Berlin-Brandenburg-schlesische Oberlausitz und Privatdozentin an der Theologischen Fakultät der Humboldt-Universität zu Berlin.

https://orcid.org/0000-0002-5502-4558

Lena Nogossek Geboren 1989; 2009-16 Studium der Kulturwissenschaft und Ev. Theologie; seit 2013 Mitarbeiterin an der Theologischen Fakultät der Humboldt-Universität zu Berlin.

Jetzt bestellen:

https://mohrsiebeck.com/buch/jesus-handbuch-9783161554094?no_cache=1

order@mohrsiebeck.com

Telefon: +49 (0)7071-923-17

Telefax: +49 (0)7071-51104 